professor of pharmacology, School of Tropical Medicine, Calcutta; (13) Psychology, Dr. G. Bose, University College of Science, 92 Upper Circular Road, Calcutta. The main part of the British Association delegation will arrive at Bombay on December 16, and will tour Northern India before attending the Congress. The address of the General Secretary of the Indian Science Congress Association is c/o The Geological Survey of India, 27 Chowringhee, Calcutta.

\section{Lubrication and Lubricants}

THE Journal of the Institution of Mechanical Engineers of May describes the arrangements which have been made for a general discussion on lubrica. tion and lubricants which is to take place at the Institution on October 13-15. The executive committee, with Dr. H. J. Gough as chairman, desires to make this an opportunity to review the present state of knowledge on the subject by means of contributions from those who are especially qualified to discuss the major problems, the immediate objects being to establish a correlation between theory, academic research and trade practice, to elucidate the methods of design of bearings, to obtain current views upon bearing metals and to explore the significance of laboratory tests. In connexion with the meetings, it is proposed to organize an exhibition. In view of the importance of the subject, the active co-operation of other societies has been invited and already more than forty British and foreign institutions have accepted. The programme, in its present state, contains about a hundred papers divided into four distinct groups dealing respectively with journal and thrust bearings, engine lubrication, industrial applications, and properties and testing, each of which has been split up into appropriate sections and subsections. From these it would appear that no aspect of the widespread applications of lubrication has been lost sight of, and besides those problems and considerations associated with all classes of machinery, such cases as those arising in connexion with wiredrawing, wire rope transmission, chains, metal pressings, cutting fluids and the like are to be subjects of discussion. In the group comprising properties and testing, the papers will deal with three main sections under the headings friction and wear, physical properties, and chemical properties. The papers already promised are being prepared by leading authorities on their subjects, and it is desired that further offers to contribute to the discussion should be addressed to the secretary, The Institution of Mechanical Engineers, Storey's Gate, London, S.W.1.

\section{Sphinx}

THE problem of publishing results too specialized for such a journal as NATURE and not substantial enough for the proceedings of a learned society, which nevertheless have some permanent value and deserve to be recorded, is difficult. Many a pertinent observation must be buried in field-club notes in the files of local weeklies, and a glance at the pages of Dickson's "History of the Theory of Numbers" will suggest the magnitude of the task of the conscientious historian in any subject in which isolated statements can be significant. These remarks are prompted by the appearance recently in Sphinx, a monthly de. voted to mathematical recreations and published privately by M. Kraitchik, Institut des Hautes Etudes de Belgique, 65 rue de la Concorde, Bruxelles, of an article "On Hyper-Exponential Numbers", in which Mr. H. J. Woodall, formerly associated with Lieut.-Colonel Cunningham in a series of extensive computations, discusses some sequences which resemble the sequence of functions forming the exponential scale but replace the $e$ of the analyst by an integer. Mr. Woodall gives specimens of classification, determines a few orders of magnitude, and finds a few inequalities. No better medium $\mathrm{c} f$ publication exists for such work, but the fact remains that a future investigator is much more likely to repeat Mr. Woodall's calculations than to discover that the work has been done.

\section{Bleaching of Wood-pulp}

IN connexion with the article on "Wood Pulp and the Future" (see Nature, 139, 867, May 22, 1937) we have received a letter from the Development and Research Department of the Pennsylvania Salt Manufacturing Co., 1000 Widener Building, Philadelphia, pointing out that the use of an emulsion of chlorine in water in multi-stage bleaching processes is the result of investigations made by this Company and is patented in twelve countries. It may be stated that the writer of the article was fully aware of the part played by the organization concerned in developing this particular method of bleaching. In a short survey covering such a wide field, however, it was not considered necessary to give references to the work of individual firms ; in the case of bleaching processes in fact, this would indeed have been a particularly difficult matter, owing to the large number involved.

\section{Genetical Society}

THE summer meeting of the Genetical Society was held at the Courtauld Genetical Laboratory, Inner Circle, 'Regent's Park, on August 10. The demon. strations shown by Prof. R. Ruggles Gates and his staff included the following : many new. species and varieties of AEnothera, in connexion with the genetic survey of the genus in eastern Canada; Indian and Japanese varieties of rice, with X-ray mutations, autotriploid and allotriploid progenies, showing an inverse relation between the number of extra chromosomes and the size of the plants ; a cytological survey of various species of the Phalarideæ; cytogenetical studies of Cicer arietinum; experiments with the growth of pollen tubes in cotton plants, and a chromosome survey of various Malvaceæ.

\section{Research on Mental Disease}

THe annual report for 1936-37 of the Birmingham Joint Board of Research for Mental Disease consists entirely of pathological laboratory findings. Fifty-six named varieties of micro-organism are described as 